\title{
3 Research Square \\ Kinesin superfamily protein 21B acts as an oncogene in non-small cell lung cancer
}

\section{Zhi-Gang Sun}

Jinan Central Hospital Affiliated to Shandong University

\section{Feng Pan}

Jinan Central Hospital Affiliated to Shandong University

Jing-Bo Shao

Jinan Central Hospital Affiliated to Shandong University

Qian-Qian Yan

Jinan Central Hospital Affiliated to Shandong University

Lu Lu

Jinan Central Hospital Affiliated to Shandong University

Nan Zhang ( $\sim$ zlkzn2016@126.com )

jinan central hospital https://orcid.org/0000-0002-6744-2810

\section{Primary research}

Keywords: non-small cell lung cancer, KIF21B, proliferation, oncogene

Posted Date: June 4th, 2020

DOI: https://doi.org/10.21203/rs.3.rs-20245/v2

License: (c) (1) This work is licensed under a Creative Commons Attribution 4.0 International License. Read Full License

Version of Record: A version of this preprint was published at Cancer Cell International on June 12th, 2020. See the published version at https://doi.org/10.1186/s12935-020-01323-7. 


\section{Abstract}

Background: Kinesin superfamily proteins (KIFs) serve as microtubule-dependent molecular motors, and are involved in the progression of many malignant tumors. In this study, we aimed to investigate the expression pattern and precise role of kinesin family member 21B (KIF21B) in non-small cell lung cancer (NSCLC).

Methods: KIF21B expression in 72 cases of NSCLC tissues was measured by immunohistochemical staining (IHC). We used shRNA-KIF21B interference to silence KIF21B in NSCLC H1299 and A549 cells and normal lung epithelial bronchus BEAS-2B cells. The biological roles of KIF21B in the growth and metastasis abilities of NSCLC cells were measured by Cell Counting Kit-8 (CCK8), colony formation and Hoechst 33342/PI, wound-healing, and Transwell assays, respectively. Expression of apoptosis-related proteins was determined using western blot. The effect of KIF21B on tumor growth in vivo was examined using nude mice model.

Results: KIF21B was up-regulated in NSCLC tissues, and correlated with pathological lymph node and pTNM stage, its high expression was predicted a poor prognosis of patients with NSCLC. Silencing of KIF21B mediated by lentivirus-delivered shRNA significantly inhibited the proliferation ability of $\mathrm{H} 1299$ and A549 cells. KIF21B knockdown increased apoptosis in H1299 and A549 cells, down-regulated the expression of Bcl-2 and up-regulated the expression of Bax and active Caspase 3. Moreover, KIF21B knockdown decreased the level of phosphorylated form of Akt (p-Akt) and Cyclin D1 expression in H1299 and A549 cells. In addition, silencing of KIF21B impeded the migration and invasion of H1299 and A549 cells. Further, silencing of KIF 21B dramatically inhibited xenograft growth in BALB/c nude mice. However, silencing of KIF21B did not affect the proliferation, migration and invasion of BEAS-2B cells.

Conclusions: These results reveal that KIF21B is up-regulated in NSCLC and acts as an oncogene in the growth and metastasis of NSCLC, which may function as a potential therapeutic target and a prognostic biomarker for NSCLC.

\section{Background}

As an important part of the cytoskeleton, the dynamic behavior of microtubules plays an important function in many biological processes, including cell motility, intracellular trafficking and mitotic spindle formation. Recently, emerging studies has reported that abnormal changes in microtubule dynamics are frequently observed during cancer cell divisions, leading to chromosomal instability, aneuploidy or drug resistances [1]. Currently, some effective anti-cancer drugs are microtubule interfering drugs such as the taxanes [2]. However, despite all these studies, the precise role of microtubules in the development of cancer is still poorly studied.

Kinesin superfamily proteins (KIFs) serve as microtubule-dependent molecular motors, play a fundamental role in cellular functions though transport certain cellular proteins, macro-molecules, and organelles $[3,4]$. Recently, it has been reported that KIFs functions in mitotic cell division by participating 
in chromosomal and spindle movement, indicating that deregulation of KIFs may be associated with tumorigenesis [5-7]. Kinesin family member 21B (KIF21B), located at 1q32.1, is the motor of Kinesin-4 motor, and contains a motor domain, a stalk and a tail domain which binds to microtubules. It has been confirmed that KIF21B, a classic kinesin protein, could function as a regulator of microtubule dynamics [8]. It could inhibit the growth of microtubules through its tail domain, acting as a potential microtubulepausing factor $[9,10]$. As well known, microtubules and kinesin proteins play critical roles in intercellular signal transduction, malignancy, tumor progression and metastasis [11]. Arai et al. showed that high KIF21B expression was correlated with poor disease-free survival rate of prostate cancer patients [12]. Zhao et al. revealed that KIF21B expression was up-regulated and associated with prognosis in hepatocellular carcinoma [13]. However, the precise role of KIF21B in carcinogenesis of non-small cell lung cancer (NSCLC) remains unclear.

In this study, we aimed to detect the expression of KIF21B in NSCLC, and investigate its functional role in the progression of NSCLC. Our data demonstrated that KIF21B was up-regulated in NSCLC, and high KIF21B expression was associated with advanced clinicopathologic features of NSCLC. Importantly, KIF21B was an independent factor for the 5-year survival rate of NSCLC patients. The loss-of-function experiments suggested that KIF21B exerted an oncogenic role in the growth and invasion of NSCLC cells.

\section{Material And Method}

\section{Patients and Tissue specimens}

A total of NSCLC samples $(n=72)$ and adjacent normal tissues $(n=20)$ in the study were collected from NSCLC patients enrolled in Jinan Central Hospital from January 2011 to December 2014. The inclusion criteria of the samples in this study were as follows: 1) patients undergo radical surgery with no seriously surgical contraindications, 2) stage I-IIla NSCLC patients, and 3) No radiotherapy or chemotherapy before surgery.

\section{Follow-up}

After operation, 62 patients had chemotherapy, 27 patients had radiotherapy, and 32 patients received therapy of EGFR-TKI. We recorded the location and tumor recurrence time, and survival was measured.

\section{Cell culture and infection}

The human NSCLC cell lines H1299 and A549 and normal lung epithelial bronchus BEAS-2B cells were obtained from Chinese Academy of Sciences Cell Bank (Shanghai, China). H1299 and A549 cells were cultured in DMEM (Hyclone, USA) supplementing with 10\% FBS (Gibco, Thermo Fisher, USA), penicillin $\left(100 \mathrm{U} / \mathrm{mL}\right.$; Sigma Aldrich, Germany) and streptomycin $\left(0.1 \mathrm{mg} / \mathrm{ml}\right.$; Sigma Aldrich) at $37^{\circ} \mathrm{C}$. BEAS-2B cells were cultured in RPMI 1640 (Gibco, USA) supplementing with 5\% FBS. The recombinant lentiviruses packaged with shRNA-KIF21B were obtained from Genechem (Shanghai Genechem Co., Ltd.). The target 
sequence of KIF21B was as follows $₫ 5^{\prime}-G G A$ GCT GAT GGA GTA TAA G-3' $₫$ The sequence of shRNA-NC control was as follows $₫ 5^{\prime}-$ TTC TCC GAA CGT GTC ACG T-3'.

\section{Immunohistochemistry(IHC)}

The paraffin-embedded samples were sectioned at $4 \mu \mathrm{m}$ and the expression of KIF21B was evaluated by immunohistochemistry using the EliVisionTMplus kit (Maixin, Fuzhou, China) with anti-KIF21B antibody (ab121931; Abcam, UK) according to the instructions provided. Two observers who were blinded with respect to the information of patients and tumor grade were performed quantification. In each case, 10 fields were randomly selected under the light microscope, and the expression level of KIF21B was evaluated by staining intensity and proportion of positive stained cells. The staining intensity was scored strong staining (3), moderate staining (2), weak staining (1) and no staining (0). The proportion of positive stained cells was graded $0-10 \%(0), 11-25 \%(1), 26-50 \%(2), 51-75 \%(3)$, and $>75 \%$ (4). The multiplication of the scores of the two groups was the final score of the expression level of KIF21B, and the score less than or equal to 6 was the low expression group, and the score more than 6 was the high expression group $[14,15]$.

\section{Reverse Transcription Polymerase Chain Reaction (RT-PCR)analysis}

We extracted total RNA from cells by an RNA extraction kit (CWBIO, Beijing, China). The reverse transcription was then conducted using a HiFiScript cDNA Synthesis Kit (CWBIO). KIF21B mRNA expression was measured by a SYBR Premix Ex Taq II kit (Takara, Japan). The obtained data was analyzed using $2-\Delta \triangle C \mathrm{Ct}$ method. $\beta$-actin was used as the control. The primers used in this study were synthesized by GENEWIZ (Suzhou, China) and as follows: KIF21B forward, 5'-CGA GGA GAC GGA TGA GAA CG-3'; reverse, 5'-CCA CCA GGC TCT CTT CAC TG-3'; $\beta$-actin forward, 5'-CCC GAG CCG TGT TTC CT3', reverse, 5'-GTC CCA GTT GGT GAC GAT GC-3'.

\section{Western blot}

After being infected for 72h, cells were lysed in RIPA Buffer (CWBIO) and quantified using a BCA kit (CWBIO). Proteins were separated with 10\% SDS-PAGE gel and then electrotransferred to PVDF membranes (Millipore, MA, USA). Subsequently, we blocked the membranes in $5 \%$ nonfat milk for $1 \mathrm{~h}$, and then incubated them with primary antibodies at $4^{\circ} \mathrm{C}$ overnight. After that, the membranes were incubated with secondary antibodies (dilution, 1:3000; Proteintech Group) for $1 \mathrm{~h}$ at room temperature. The protein signals were developed using an ECL kit (CWBIO) and analyzed using Image J software (NIH, USA) [16].

\section{Cell Counting Kit-8 (CCK8) assay}

$1 \times 10^{3}$ cells transfected with shRNAs were seeded in a 96 -well plate at $37^{\circ} \mathrm{C}$ for $1,24,48$ and $72 \mathrm{~h}$, respectively. Cells were then incubated with $10 \mu \mathrm{l}$ of CCK8 solution (Solarbio Science \& Technology, Beijing, China) for another $1 \mathrm{~h}$ at $37^{\circ} \mathrm{C}$. Finally, a plate reader determined the OD value at $450 \mathrm{~nm}$. 
Cells infected with shRNA-KIF21B lentivirus (200 cells/well) were plated in a 6-well plate and cultured for 1-2 weeks. After being fixed with $4 \%$ paraformaldehyde for $30 \mathrm{~min}$, the cells were stained with $0.1 \%$ crystal violet for another 30 min. Finally, we counted the number of colonies.

\section{Hoechst 33342/PI assay}

Cells infected with shRNAs $\left(1 \times 10^{3}\right.$ cells/well) were cultured in a well plate for $24 \mathrm{~h}$. Then, we co-stained the cells with Hoechst $33342(10 \mu \mathrm{g} / \mathrm{mL}$; Beyotime, Shanghai, China) and PI (5 $\mathrm{gg} / \mathrm{mL}$; Beyotime) for 15 min. After washing with PBS for 3 times, cell fluorescence was observed and captured under a fluorescence microscope.

\section{Wound-healing assay}

Following infection of $24 \mathrm{~h}$, the cells $\left(5 \times 10^{5}\right.$ cells/well) were cultured in a 6 -well plate for $12 \mathrm{~h}$. A wound was generated using a pipette tip in each well. After $24 \mathrm{~h}$ of culture, the wound closure was measured.

\section{Transwell assay}

Transwell chambers (Millipore) coated with Matrigel was carried out to assess cell invasion, whereas cell migration assay did not add Matrigel. We plated about $1 \times 10^{5}$ lentivirus-infected cells in the upper chamber; the lower chamber was filled with medium containing FBS. Subsequent to a period of $24 \mathrm{~h}$, we used cotton swabs to wipe out the cells that were not through the membrane. We fixed the migrated or invaded cells in $4 \%$ paraformaldehyde for $30 \mathrm{~min}$, and then stained them with $0.1 \%$ crystal violet for another $20 \mathrm{~min}$. The number of migrated or invaded cells was quantified under a microscope (magnification, $\times 100$ ) in five random microscope fields.

\section{In vivo tumorigenic assay}

According to the $\mathrm{NIH}$ guidelines, animal procedures were carried out. Ten BALB/c female nude mice (20 \pm 2 g) were upraised in barrier facilities on a 12 hours light/dark cycle and randomly divided into 2 groups $(n=5)$. H1299 cells were infected with lentivirus-shRNA-KIF21B or lentivirus-shRNA-control for $48 \mathrm{~h}$. The $\mathrm{H} 1299$ cells $\left(5 \times 10^{6}\right)$ were subcutaneously implanted in the right axils of nude mice. All nude mice were euthanized five weeks following injection, the tumors were removed and their weights were measured.

\section{Statistical analysis}

The measurement data were presented as Mean \pm SD and analyzed using Student's t-test or one-way ANOVA. The enumeration data were analyzed using Fisher's exact probability test. The Kaplan-Meier with log-rank test was performed for survival rate. Cox proportional hazard model was performed for multivariate analysis. We used SPSS (IBM SPSS Statistics 25, USA) to perform for statistical analysis. And $\mathrm{P}<0.05$ was regarded as significant difference.

\section{Results}




\section{KIF21B is up-regulated in NSCLC tissues}

As indicated by IHC assay (Figure 1), we found that the positive KIF21B signal was mainly located in cytoplasm. KIF21B expression in the NSCLC tissues (44/72, 61.1\%) was significantly higher than that in adjacent normal lung tissues $(0 / 20,0.0 \%)(P<0.01$, Figure $1 \mathrm{~A}-\mathrm{H})$. The high expression of KIF21B was correlated with $\mathrm{pN}(P<0.05)$ and pTNM stage $(P<0.05)$ (Table 1). Moreover, the 5 -year survival rate of the 72 cases of NSCLC was $40.3 \%$, which was significantly correlated with tumor differentiation $(P<0.05), \mathrm{pN}$ $(P<0.01)$, and pTNM stage $(P<0.01)$ (Figure 2A-D and Table 2). Importantly, NSCLC patients with higher KIF21B expression had poor prognosis $(P<0.01)$ (Figure 2E and Table 2). Further, $\mathrm{pN}$ and KIF21B were independent factors for the NSCLC patients' 5-year survival rate (Table 3).

\section{Silencing of KIF21B impedes the proliferation of NSCLC cells}

Given the up-regulation of KIF21B expression in NSCLC tissues, in order to further investigate its biological function in NSCLC, we silenced the expression of KIF21B in NSCLC cell lines and normal BEAS$2 B$ cells by infecting with shRNA-KIF21B-lentiviral. As indicated in Figure $3 A$ and $B$, the expression of KIF21B was obviously down-regulated in $\mathrm{H} 1299$ and $\mathrm{A} 549$ cells at both mRNA and protein levels. The expression of KIF21B was also reduced by sh-KIF21B in BEAS-2B cells (Figure $3 C$ and D). CCK8 assay showed that KIF21B knockdown reduced the proliferation abilities of both $\mathrm{H} 1299$ and $A 549$ cells (Figure $3 \mathrm{E}$ and F). However, silencing of KIF21B did not affect the proliferation of BEAS-2B cells (Figure 3G). Further, we performed colony formation assay to assess the colony-forming abilities of H1299 and A549 cells after KIF21B was silenced. As shown in Figure $3 \mathrm{H}$ and $\mathrm{I}$, the results further confirmed that silencing of KIF21B inhibited the colony-forming abilities of H1299 and A549 cells, but not BEAS-2B cells.

\section{Knockdown of KIF21B promotes apoptosis and inhibits activation of the Akt signaling pathway in NSCLC cells}

After being infected with sh-KIF21B for $72 \mathrm{~h}$, cell apoptosis was determined using the Hoechst/PI assay. A significant increase in the percentage of apoptotic cells was observed in KIF21B-silenced cells compared with the NC group (Figure 4A). Moreover, western blot assay revealed that the expression of Bcl-2 was significantly decreased in both $\mathrm{H} 1299$ and A549 cells after KIF21B was silenced, while the expression levels of Bax and active Caspase3 were increased (Figure 4B). But we observed no significant effect of KIF21B knockdown on the expression levels Bcl-2, Bax and active Caspase 3 in BEAS-2B cells (Figure 4C). Based on these data, silencing of KIF21B may promote apoptosis in NSCLC cells by modulating the Bcl-2/Bax expression and Caspase 3 activation.

Given the critical role of the Akt signaling pathway involved in the growth, metastasis and apoptosis of cancer cells, the effect of KIF21B on this signaling pathway was evaluated. Western blot demonstrated that KIF21B knockdown did not affect the level of total Akt in H1299 and A549 cells, but significantly decreased the phosphorylation form of Akt (p-Akt) (Figure 4D). Consistently, the expression of downstream protein Cyclin D1 was inhibited by KIF21B knockdown in NSCLC cells (Figure 4D). However, 
there was no significant change in the expression of Akt, p-Akt and Cyclin D1 in BEAS-2B cells after KIF21B was silenced (Figure 4E).

\section{KIF21B inhibition suppresses the migration and invasion abilities of NSCLC cells}

As indicated by wound-healing assay, the migration abilities of H1299 cells were significantly impeded after KIF21B was silenced (Figure 5A). Similarly, the migration surface of A549 cells transfected with shKIF21B was also significantly decreased compared with the NC group (Figure 5A). Additionally, results of transwell assay manifested that the number of migrated cells was significantly decreased by silencing KIF21B (Figure 5B), which was further supported that KIF21B inhibition suppressed NSCLC cell migration. Moreover, in comparison with the NC group, $\mathrm{H} 1299$ and A549 cells infected with sh-KIF21B displayed a significant depression in cell invasion (Figure 5C). Interestingly, silencing of KIF21B did not affect the migration and invasion capacity of BEAS-2B cells (Figure 5D and E).

\section{Down-regulation of KIF21B inhibits xenograft growth in nude mice}

Since the effects of KIF21B on NSCLC cells had been revealed by in vitro experiments, we further investigate its effect on tumor growth in vivo. The sh-NC and sh-KIF21B H1299 cells were subcutaneously (s.c.) xenografted into the BALB/c nude mice. The tumors were excised at the end of the experiment (Figure $6 \mathrm{~A}$ and $\mathrm{B}$ ). Tumor weights were determined and plotted in Figure $6 \mathrm{C}$. Results showed that the tumor size and weight of KIF21B-silenced group were significantly lower than those of control group (Figure 6A-C).

\section{Discussion}

It is a generally acknowledged that lung cancer remains the main cause of tumor-related deaths globally, with about 1.4 million new cases diagnosed and approximately 1.6 million deaths per year worldwide $[17,18]$. NSCLC is reported to account for nearly $85 \%$ of lung cancer. Tumor metastasis is observed in $79 \%$ of patients with NSCLC, and the 5-year survival rate of advanced patients is no more than $5 \%[19,20]$. Therefore, it is urgent to search for novel and reliable targets for NSCLC therapy.

In view of the critical roles of KIFs in mitotic cell division, accumulating evidence has demonstrated that $\mathrm{KIFs}$ are involved in tumorigenesis [21,22]. For instance, previous reports revealed that up-regulation of KIF14 could promote cancer progression and metastasis, and could predict poor prognosis in gastric cancer and colorectal cancer [23, 24]. Gu et al. suggested that silencing of KIF26B suppressed breast cancer cell growth and invasion [25]. KIFC1 is also reported to be participated in the progression of NSCLC through regulation of cell proliferation and cell cycle [7]. Therefore, further understanding of the relationship between KIFs and tumor progression can provide a new perspective on the mechanism of tumorigenesis and serve effective targets for cancer therapy. Herein, our data highlight the oncogenic role of KIF21B in NSCLC. 
Recently, Zhao et al. revealed that KIF21B was up-regulated in hepatocellular carcinoma tissues and cells, which was an independent risk factor for overall survival and disease-free survival in patients with hepatocellular carcinoma after hepatectomy [13]. In the present study, we determined that KIF21B was up-regulated in NSCLC tissues compared to adjacent normal tissues, which was consistent with the expression pattern of KIF21B in hepatocellular carcinoma. Moreover, high KIF21B expression was correlated with lymph node metastasis and tumor stage, predicting a poor prognosis of patients with NSCLC. Hence, based on these findings, KIF21B may function as a potential prognostic biomarker in NSCLC might be participated in the development of NSCLC.

It's well known that uncontrolled proliferation is a prominent feature of malignant tumors. Gu et al. showed that depletion of KIF26B triggers cell cycle could arrest through regulating CyclinD1 to modulate cell proliferation in mammary cancer [25]. Liu et al. showed that knockdown of KIFC1 could reduce cell proliferation and trigger G2/M phase arrest in A549 and SPC-A1 cells [7]. It has been recently reported that silencing of KIF21B could inhibit the proliferation of hepatocellular carcinoma BEL-7404 cells [13]. In this study, the loss-of-function experiments manifested that silencing of KIF21B suppressed the proliferation of $\mathrm{H} 1299$ and A549 cells, and inhibited NSCLC tumor growth in vivo, which was consistent with the proliferation promoting effect of KIF21B in hepatocellular carcinoma cells. Moreover, we observed a significant increase in apoptosis in KIF21B-silenced cells. The Bcl-2 family serves as the key regulators of intrinsic apoptotic pathway which is the main mechanism of apoptosis, and the Bcl-2/Bax is a key regulator in determining cell fate [26]. Casapse 3 is another crucial executioner in apoptosis process [27]. We found that the expression of anti-apoptotic protein $\mathrm{Bcl}-2$ was significantly downregulated after KIF21B knockdown, while the expression of pro-apoptotic proteins Bax and active Casapse 3 was up-regulated. Therefore, these data suggested that down-regulation of KIF21B might promote apoptosis in NSCLC cells by regulating Bcl-2/Bax axis and Caspase 3 activation. Importantly, silencing of KIF21B did not affect the proliferation of BEAS-2B cells, as well as the apoptosis-related signaling. Taken together, these findings indicated an oncogenic role of KIF21B in NSCLC cell proliferation and apoptosis, and regulation of apoptosis by KIF21B might be involved in its regulation of NSCLC cell growth.

It has been widely held that the Akt signaling pathway, frequently over-activated in cancers, plays a central role in regulation of cellular processes and tumor progression such as proliferation and apoptosis [28]. Targeting Akt has been considered as an important approach for cancer prevention and therapy [29]. Therefore, to preliminarily explore the mechanism by which KIF21B affected NSCLC cell proliferation and apoptosis, we examined the effect of KIF21B knockdown on the Akt signaling pathway. Our data demonstrated that knockdown of KIF21B reduced activation of the Akt signaling pathway by decreasing the level of phosphorylation form of Akt in NSCLC cells, but did not affect the Akt signaling pathway in BEAS-2B cells. Additionally, the expression of Cyclin D1, a downstream protein of the Akt signaling pathway, was down-regulated in KIF21B-silenced cells. Cyclin D1 is revealed to function as a pivotal regulator of cell cycle, promoting the transition of cells from $\mathrm{G} 1$ to $S$ phase [28]. Collectively, these results suggest that the Akt signaling pathway may be involved in the oncogenic role of KIF21B in NSCLC. Further, it is important to note that one of the leading causes of poor prognosis of cancer patients is 
tumor metastasis. Our data demonstrated that silencing of KIF21B decreased the migration and invasion of $\mathrm{H} 1299$ and A549 cells, but not BEAS-2B cells, suggesting the potential role of KIF21B in modulation of tumor metastasis. It has been confirmed that KIF26B regulates cell invasion in breast cancer through driving epithelial-mesenchymal transition (EMT) [23]. The effect of KIF21B on the EMT process in NSCLC will be further studied in our future research.

\section{Conclusion}

In summary, for the first time, the current study demonstrated that KIF21B was up-regulated in NSCLC tissues, and its high expression was associated with poor clinicopathologic features and predicts a poor prognosis in NSCLC. Moreover, our data confirm that KIF21B exerts an oncogenic role in NSCLC progression, silencing of KIF21B can suppress the growth and invasion of NSCLC cells, as well as promoting apoptosis. Therefore, KIF21B may serve as a potential prognostic biomarker and therapeutic target for NSCLC.

\section{Declarations}

\section{Acknowledgements}

Not applicable.

\section{Authors' contributions}

ZS, FP, and NZ designed the concept and experiments. All authors performed the experiments, collected the data, and analyzed the results. NZ wrote the manuscript.

\section{Funding}

The present study was funded by Jinan Science and Technology Development Program (grant no. 201907018) and Shandong Provincial Key Research and Development Program (grant no. 2017G006037).

\section{Availability of data and materials}

The data supporting the conclusions of this paper are included within the manuscript.

\section{Ethics approval and consent to participate}

The research was approved by the Research Ethics Committee of Jinan Central Hospital. All the patients signed written informed consent.

\section{Consent for publication}

The publication of this work is approved by all authors involved. 


\section{Competing interests}

The authors declare that they have no competing interests.

\section{Author details}

${ }^{1}$ Department of Thoracic Surgery, Central Hospital Affiliated to Shandong First Medical University; Jinan 250013, P.R. China. ${ }^{2}$ Department of Ethics Committee, Central Hospital Affiliated to Shandong University, Jinan 250012, P.R. China. ${ }^{3}$ Weifang Medical University, Weifang 261053, P.R. China. ${ }^{4}$ Department of Oncology, Jinan Central Hospital,Cheeloo College of Medicine, Shandong University, Jinan 250012, People's Republic of China. ${ }^{5}$ Shandong First Medical University, Jinan 250013, Shandong Province, China. ${ }^{6}$ Department of Oncology, Central Hospital Affiliated to Shandong First Medical University; Jinan 250013, P.R. China

\section{References}

1. Mohan R, Banerjee M, Ray A, Manna T, Wilson L, Owa T, et al. Antimitotic Sulfonamides Inhibit Microtubule Assembly Dynamics and Cancer Cell Proliferation. Biochemistry 2006, 45: 5440-5449.

2. Mcgrogan BT, Gilmartin B, Carney DN, Mccann A. Taxanes, microtubules and chemoresistant breast cancer. Biochim Biophys Acta 2008, 1785: 96-132.

3. Hirokawa N, Niwa S, Tanaka Y. Molecular Motors in Neurons: Transport Mechanisms and Roles in Brain Function, Development, and Disease. Neuron 2010, 68: 610-638.

4. Hirokawa N, Tanaka Y. Kinesin superfamily proteins (KIFs): Various functions and their relevance for important phenomena in life and diseases. Experimental Cell Research 2015, 334: 16-25.

5. Hirokawa N, Noda YY. Kinesin superfamily motor proteins and intracellular transport. Nature Reviews Molecular Cell Biology 2009, 10: 682-696.

6. Sharp DJ, Rogers GC, Scholey JM. Microtubule motors in mitosis. Nature 2000, 407(6800): 41.

7. Liu Y, Zhan P, Zhou Z, Xing Z, Zhu S, Ma C, et al. The overexpression of KIFC1 was associated with the proliferation and prognosis of non-small cell lung cancer. Journal of Thoracic Disease 2016, 8: 2911.

8. Muhia M, Thies E, Labonté D, Ghiretti AE, Gromova KV, Xompero F, et al. The kinesin KIF21B regulates microtubule dynamics and is essential for neuronal morphology, synapse function and learning and memory. Cell Reports 2016, 15: 968-977.

9. Labonté D, Thies E, Kneussel M. The kinesin KIF21B participates in the cell surface delivery of $\mathrm{Y} 2$ subunit-containing GABA A receptors. European Journal of Cell Biology 2014, 93: 338-346.

10. van Riel WE, Rai A, Bianchi S, Katrukha EA, Liu Q, Heck AJ, et al. Kinesin-4 KIF21B is a potent microtubule pausing factor. Elife 2017, 6: e24746.

11. He M, Subramanian R, Bangs F, Omelchenko T, Liem KF, Jr, Kapoor TM, et al. The kinesin-4 protein Kif7 regulates mammalian Hedgehog signalling by organizing the cilium tip compartment. Nat Cell 
Biol 2014, 16: 663-672.

12. Arai T, Kojima S, Yamada Y, Sugawara S, Kato M, Yamazaki K, et al. Pirin: a potential novel therapeutic target for castration-resistant prostate cancer regulated by miR-455-5p. Mol Oncol. 2019; 13: 322 -337.

13. Zhao HQ, Dong BL, Zhang M, Dong XH, He, Y, Chen SY, et al. Increased KIF21B expression is a potential prognostic biomarker in hepatocellular carcinoma. World J Gastrointest Oncol 2020, 12(3): 276-288.

14. Zhang Q, Tan XP, Yuan YS, Hu CM, He CH, Wang WZ, et al. Decreased expression of KLF6 and its significance in gastric carcinoma. Med Oncol. 2010; 27:1295-1302.

15. Zhang N, Li Z, Xiao W, Yang, Gao W, Sun ZG. KLF6-SV1 is a new prognostic biomarker in postoperative patients with non-small cell lung cancer. Cancer Manag Res. 2018; 10:3937-3944.

16. Wang D, Cai L, Tian X, Li W. MiR-543 promotes tumorigenesis and angiogenesis in non-small cell lung cancer via modulating metastasis associated protein 1. Mol Med 2020, 26(1): 44.

17. Torre LA, Bray F, Siegel RL, Ferlay J, Lortettieulent J, Jemal A. Global cancer statistics, 2012. Ca A Cancer Journal for Clinicians 2015, 65: 87-108.

18. Herbst RS, Morgensztern D, Boshoff C. The biology and management of non-small cell lung cancer. Nature 2018, 553: 446.

19. Zhu H, Chang LL, Yan FJ, Hu Y, Zeng CM, Zhou TY, et al. AKR1C1 Activates STAT3 to Promote the Metastasis of Non-Small Cell Lung Cancer. Theranostics 2018, 8: 676-692.

20. Siegel RL MK, Jemal A. Cancer statistics, 2016. CA Cancer J Clin 2016, 66(1): 7-30.

21. Huszar D, Theoclitou ME, Skolnik J, Herbst R. Kinesin motor proteins as targets for cancer therapy. Cancer \& Metastasis Reviews 2009, 28: 197-208.

22. Lucanus AJ, Yip GW. Kinesin superfamily: roles in breast cancer, patient prognosis and therapeutics. Oncogene 2018, 37(7): 833-838. .

23. Yang Z, Li C, Yan C, Li Y, Yan M, Liu B, et al. KIF14 promotes tumor progression and metastasis and is an independent predictor of poor prognosis in human gastric cancer. Biochim Biophys Acta Mol Basis Dis 2019, 1865(1): 181-192.

24. Wang ZZ, Yang J, Jiang BH, Di JB, Gao P, Peng L, et al. KIF14 promotes cell proliferation via activation of Akt and is directly targeted by miR-200c in colorectal cancer. Int J Oncol 2018, 53(5): 1939-1952.

25. Gu S, Liang H, Qi D, Mao L, Mao G, Qian L, et al. Knockdown of KIF26B inhibits breast cancer cell proliferation, migration, and invasion. Oncotargets \& Therapy 2018, 11: 3195-3203.

26. Liu G, Pei F, Yang F, Li L, Amin AD, Liu S, et al. Role of Autophagy and Apoptosis in Non-Small-Cell Lung Cancer. International Journal of Molecular Sciences 2017, 18: 367.

27. Zheng L, Zheng J, Wu LJ, Zhao YY. Julibroside J 8 -induced HeLa cell apoptosis through caspase pathway. Journal of Asian Natural Products Research 2006, 8: 457-465. 
28. Kwong LN, Davies MA. Navigating the therapeutic complexity of PI3K pathway inhibition in melanoma. Clinical Cancer Research An Official Journal of the American Association for Cancer Research 2013, 19: 5310-5319.

29. Song M, Bode AM, Dong Z, Lee MH. AKT as a Therapeutic Target for Cancer. Cancer Res 2019, 79(6): 1019-1031.

\section{Tables}

Table 1

Correlation between KIF21B expression and clinicopathological features of the patients with non-small cell lung cancer

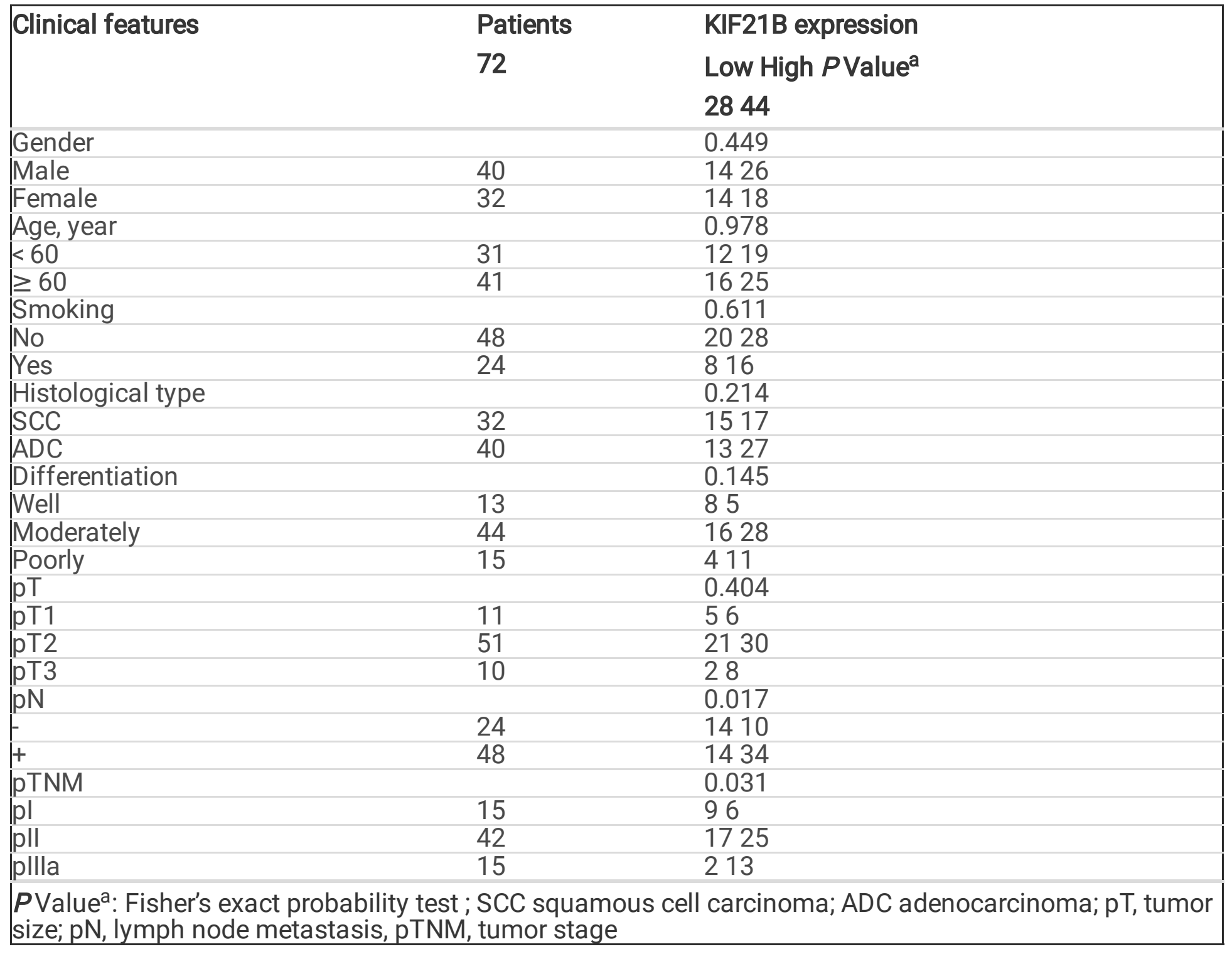


Table 2

Univariate analysis with respect to 5-year survival of the patients with non-small cell lung cancer

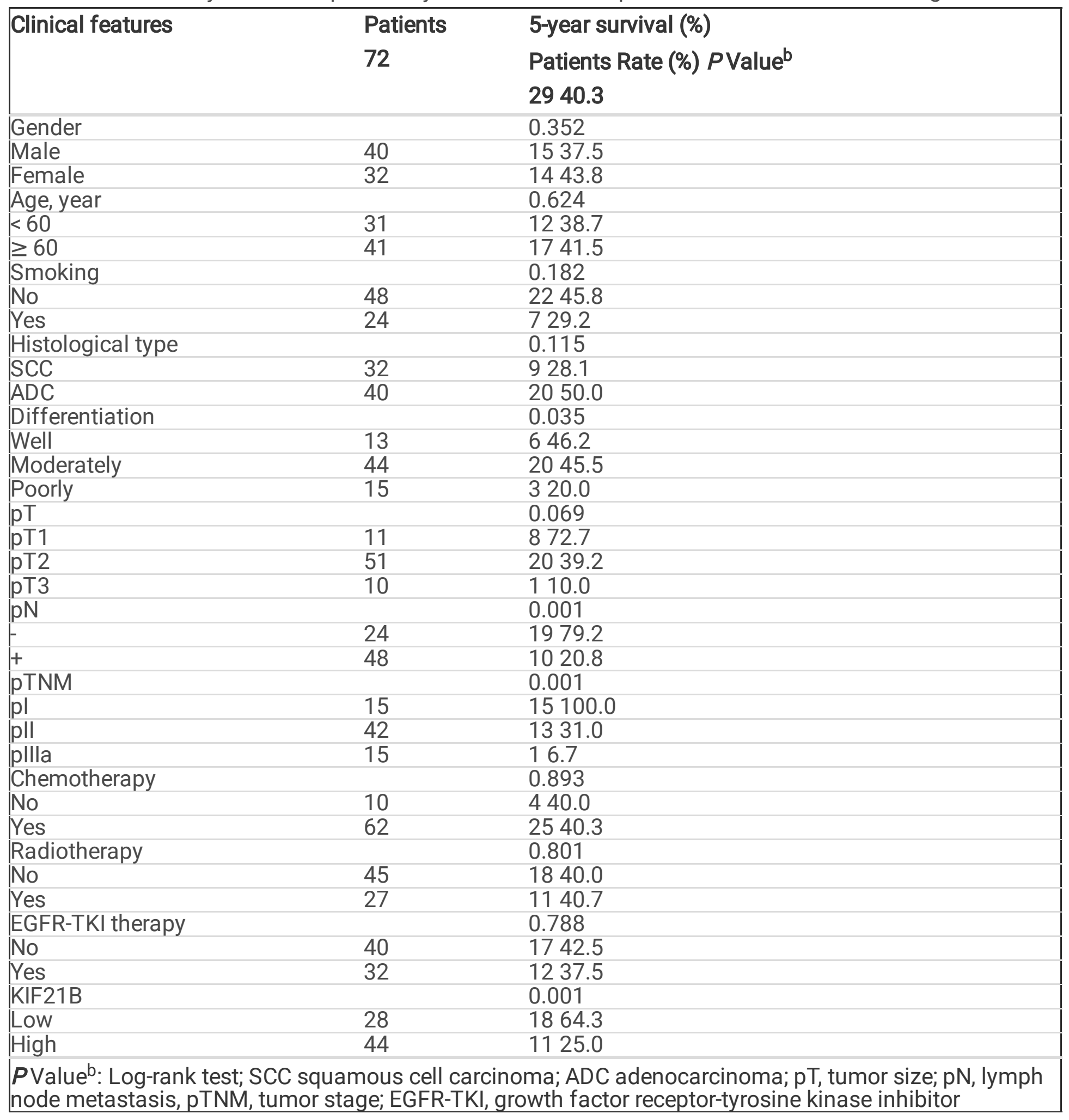


Table 3

Results of cox regression multivariate 5-year survival analysis of the patients with non-small cell lung cancer

\begin{tabular}{|lllllll|}
\hline & B & SE & Wald & P & HR & 95.0\% Cl for HR \\
\hline Gender & 0.573 & 0.409 & 1.968 & 0.161 & 1.774 & $0.796 \sim 3.951$ \\
Age & -0.332 & 0.372 & 0.796 & 0.372 & 0.718 & $0.346 \sim 1.488$ \\
Somking & -0.079 & 0.467 & 0.029 & 0.865 & 0.924 & $0.370 \sim 2.307$ \\
Histological type & -0.755 & 0.436 & 1.335 & 0.248 & 1.424 & $0.782 \sim 2.594$ \\
Differentiation & 0.353 & 0.306 & 2.757 & 0.097 & 1.707 & $0.908 \sim 3.209$ \\
\hline PT & 0.455 & 0.390 & 1.357 & 0.244 & 1.576 & $0.733 \sim 3.387$ \\
pN & 1.948 & 0.656 & 8.805 & 0.003 & 7.011 & $1.937 \sim 25.379$ \\
pTNM & 0.055 & 0.401 & 0.019 & 0.890 & 1.057 & $0.482 \sim 2.318$ \\
Chemotherapy & -0.749 & 0.576 & 1.692 & 0.193 & 0.473 & $0.153 \sim 1.462$ \\
Radiotherapy & 0.257 & 0.381 & 0.455 & 0.500 & 1.293 & $0.613 \sim 2.726$ \\
EGFR-TKI therapy & -0.072 & 0.503 & 0.020 & 0.8587 & 0.931 & $0.348 \sim 2.493$ \\
\hline KIF21B & 0.900 & 0.439 & 5.081 & 0.0040 & 2.460 & $1.041 \sim 5.811$ \\
\hline B, regression coefficient; SE, standard error; Wald, Wald value; HR, hazard ratio; Cl , confidence interval.
\end{tabular}

\section{Figures}
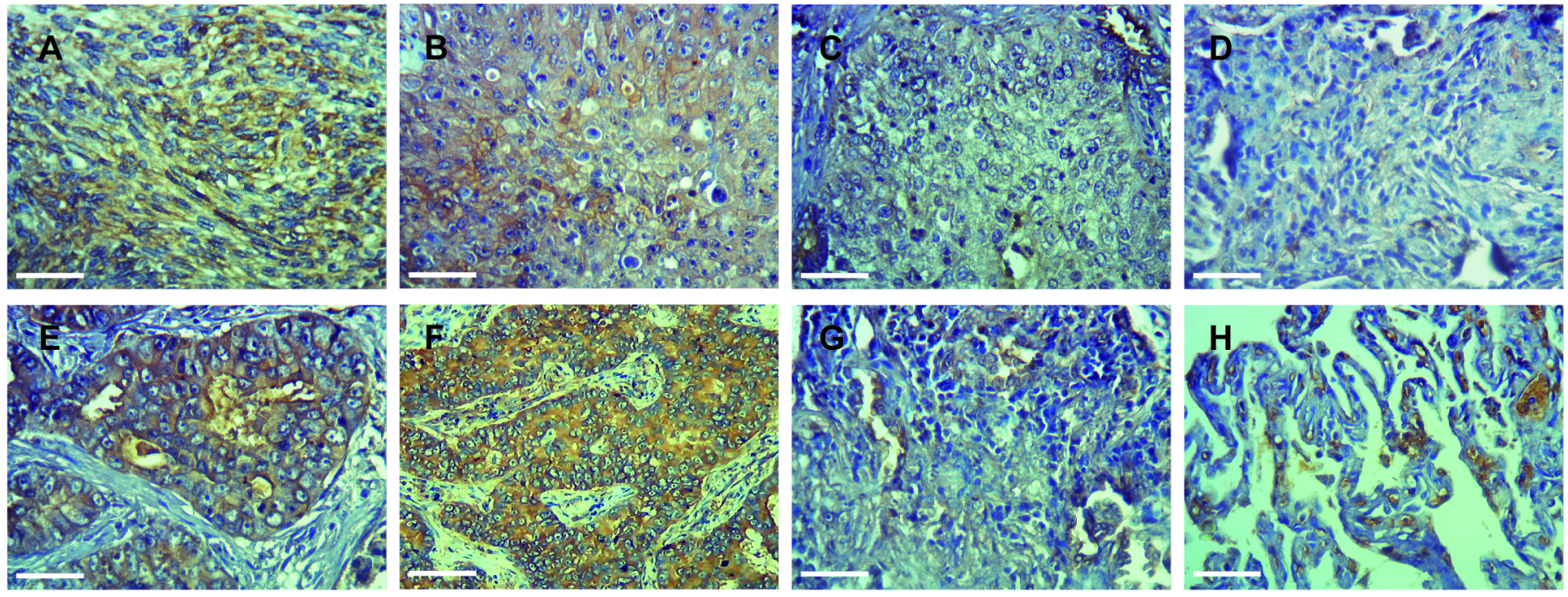

Figure 1

Immunohistochemical staining of lung cancer tissue sections demonstrating KIF21B (original magnification $\times 400)$. (A) and (B) Lung squamous cell carcinoma specimen with high expression of KLF6SV1. (C) Lung squamous cell carcinoma specimen with low expression of KLF6-SV1. (D) The corresponding normal lung tissue specimen with no KLF6-SV1 expression (Control). (E) and (F) Lung adenocarcinoma specimen with high expression of KLF6-SV1. (G) Lung adenocarcinoma specimen with low expression of KLF6-SV1. (H) The corresponding normal lung tissue specimen with no KLF6-SV1 expression (Control). The scale bar was $50 \mu \mathrm{m}$. 

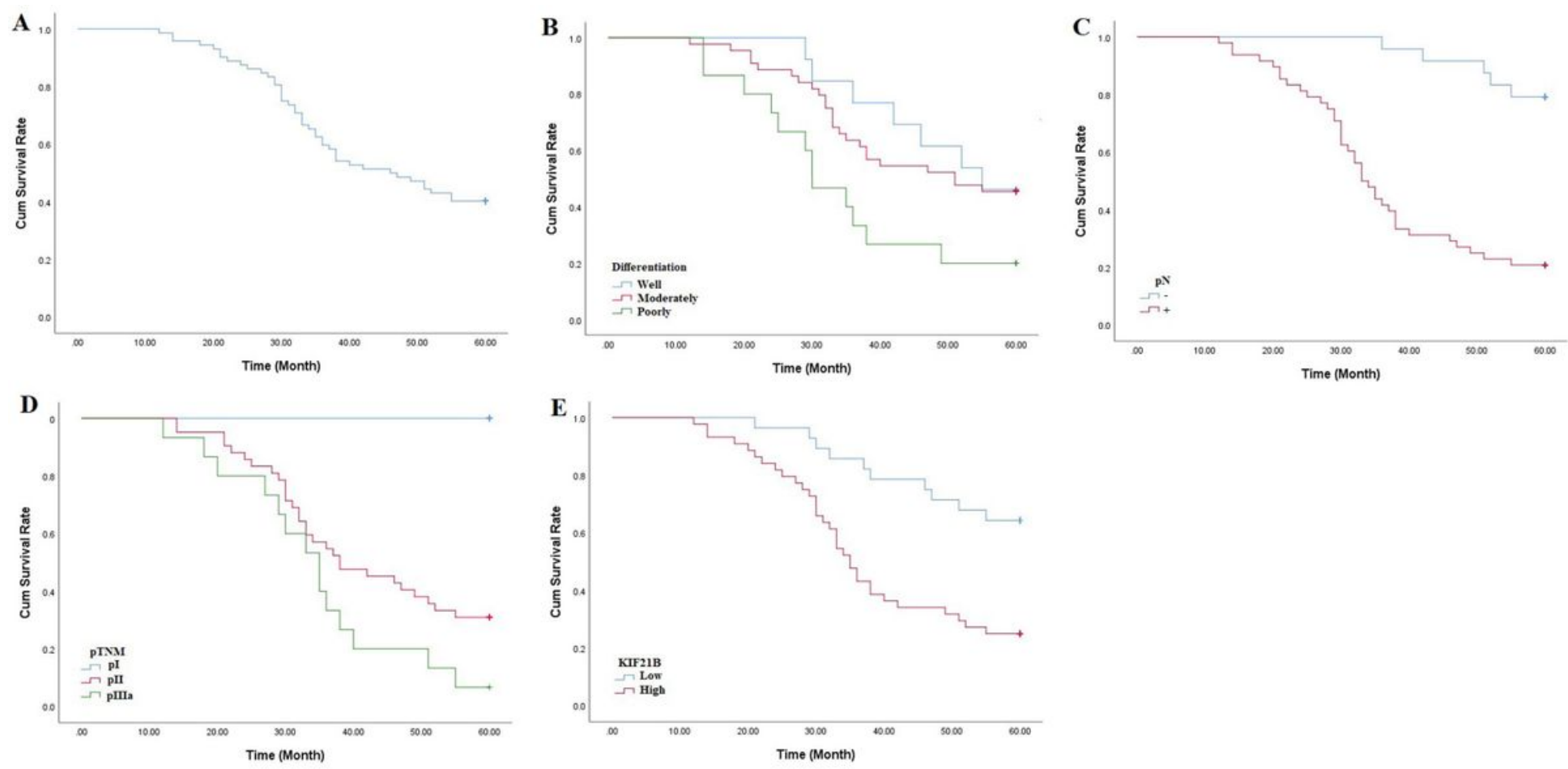

Figure 2

(A) The Kaplan-Meier survival curve of 72 cases of NSCLC patients. (B) Survival curves of NSCLC patients with tumor differentiation. (C) Survival curves of NSCLC patients with negative or positive pN. (D) Survival curves of NSCLC patients with different pTNM. (E) Survival curves of NSCLC patients with low or high expression of KIF21B expression. 
A

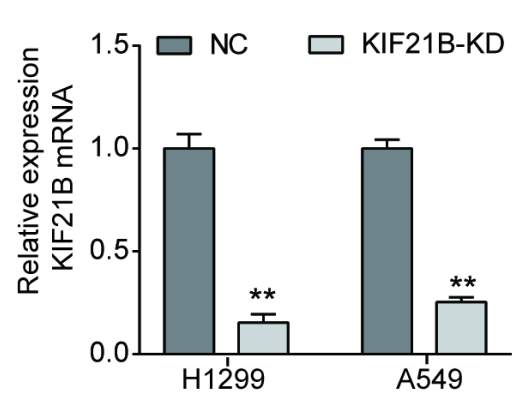

E

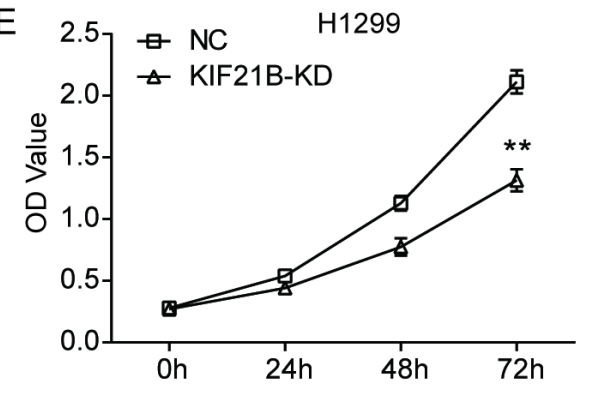

H

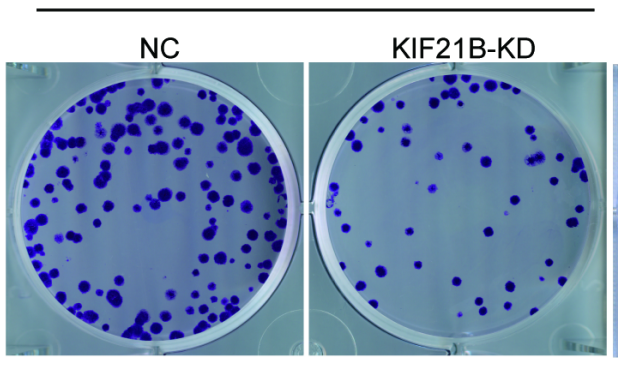

$\mathrm{H} 1299$

I

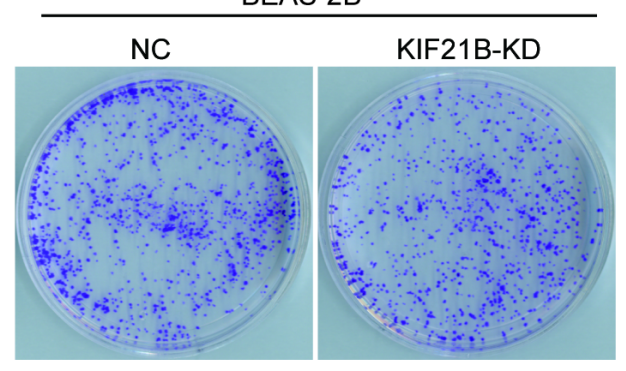

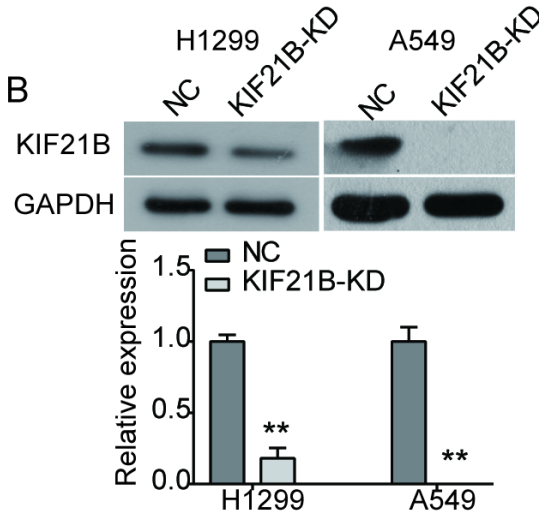

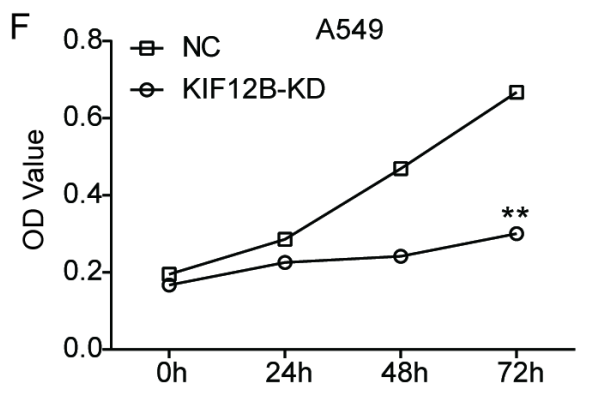

G

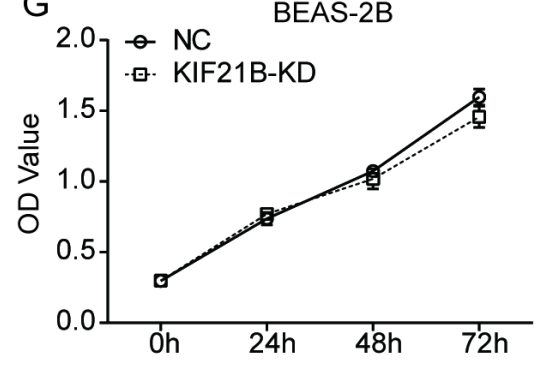

A549
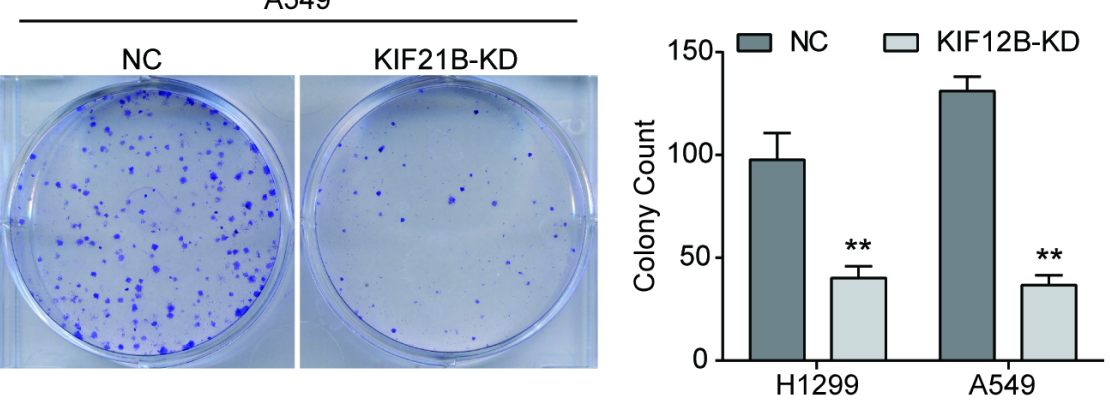

\section{Figure 3}

Knockdown of KIF21B inhibits the proliferation and colony formation abilities of NSCLC cells. H1299 and A549 cells were infected with shRNA-control lentivirus or sh-KIF21B lentivirus, as well as BEAS-2B cells. (A) qRT-PCR analysis of the expression of KIF21B mRNA in H1299 and A549 cells. (B) The expression of KIF21B protein in H1299 and A549 cells infected with sh-KIF21B lentivirus was detected by western blot. (C) qRT-PCR analysis of the expression of KIF21B mRNA in BEAS-2B cells. (D) The expression of KIF21B protein in BEAS-2B cells was detected by western blot. (E), (F) and (G) CCK8 assay was performed to 
examine the viability of H1299 (E), A549 (F) and BEAS-2B (G) cells after indicated transfection. (H) Colony formation abilities of H1299 and A549 cells were assessed by colony formation assay. (I) Colony formation ability of BEAS-2B cells was assessed by colony formation assay. NC, cells were infected with shRNA-control lentivirus; KIF21B-KD, cells were infected with sh-KIF21B lentivirus (KIF21B-knockdown). Data are presented as the mean \pm standard deviation. Results were obtained in three replicates. ${ }^{*} \mathrm{P}<0.01$.

A
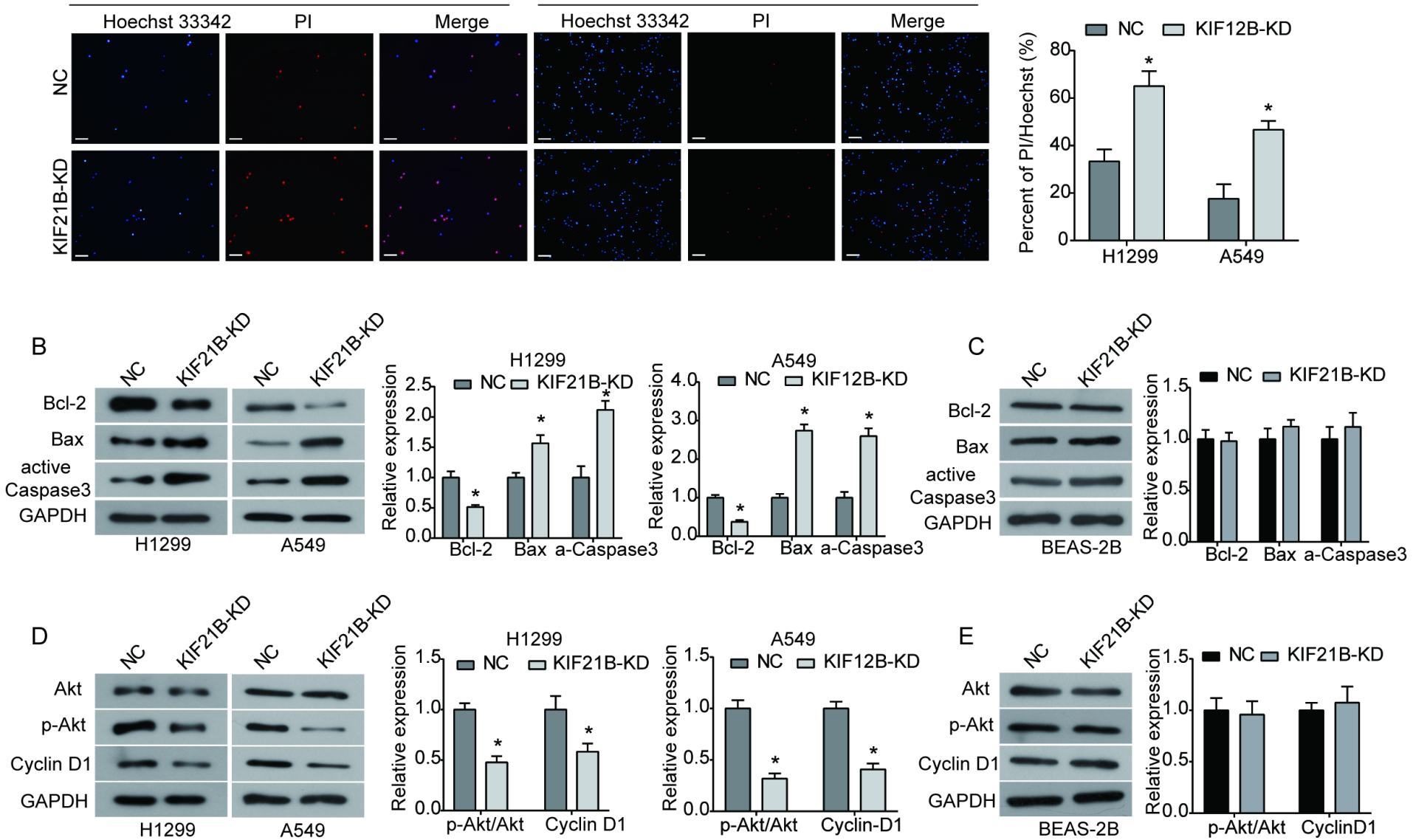

\section{Figure 4}

Knockdown of KIF21B promotes apoptosis in NSCLC cells. (A) After being infected with sh-KIF21B lentivirus, Hoechst 33342/PI assay was used to assess cell apoptosis in H1299 and A549 cells. The scale bar was $100 \mu \mathrm{m}$. (B) The expression of apoptosis-related proteins (Bcl-2, Bax and active Caspase 3) was detected by western blot in H1299 and A549 cells after indicated transfection. (C) The expression of apoptosis-related proteins was detected by western blot in BEAS-2B cells. (D) The expression of key components of the Akt signaling pathway (Akt, p-Akt and Cyclin D1) was examined using western blot in H1299 and A549 cells. (E) The expression of key components of the Akt signaling pathway was examined using western blot in BEAS-2B cells. Data are presented as the mean \pm standard deviation. Results were obtained in three replicates. ${ }^{*} \mathrm{P}<0.05$. 
A
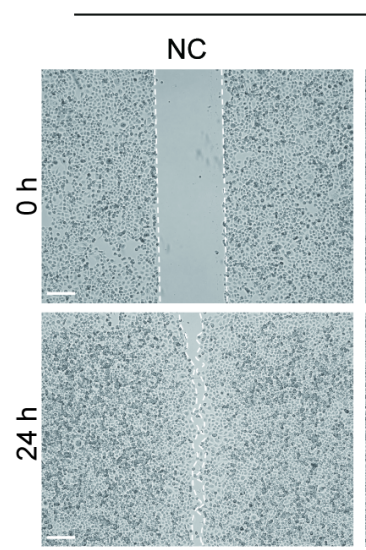

H1299
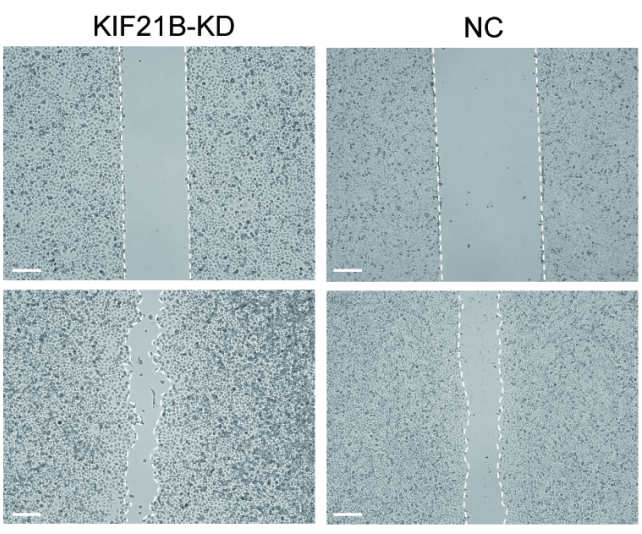

A549

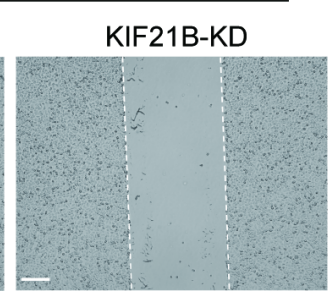

B H1299
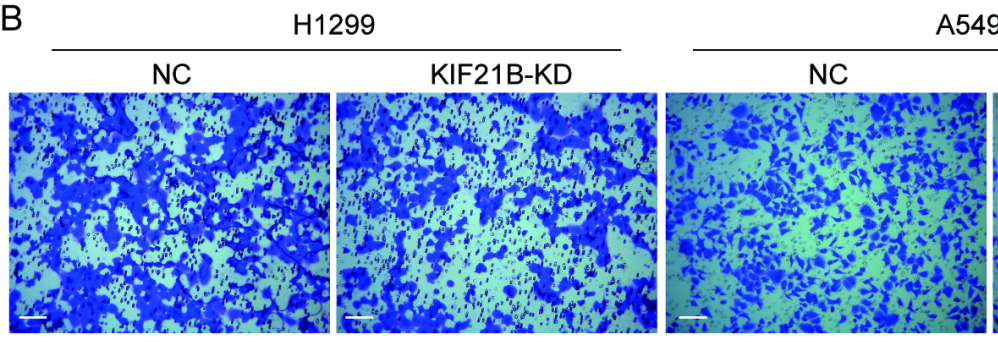

A549

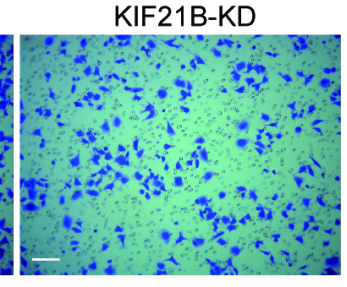

C

H1299
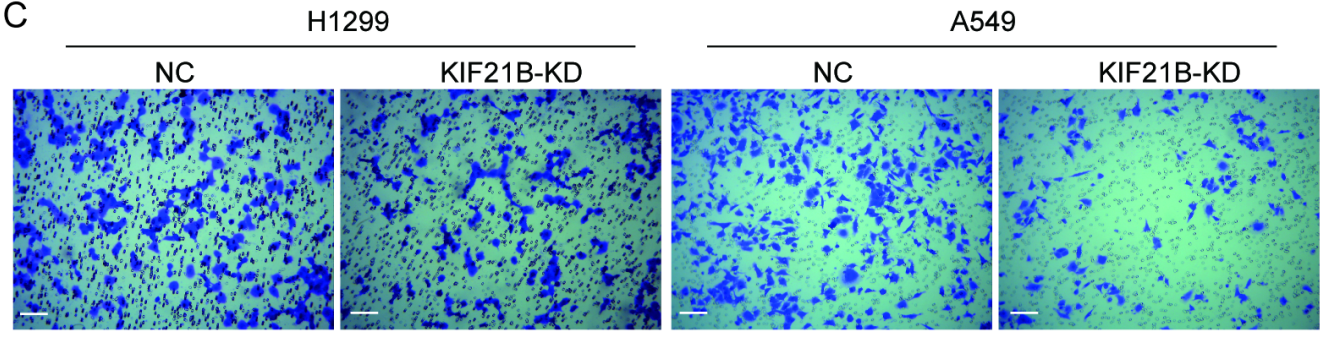

D

BEAS-2B
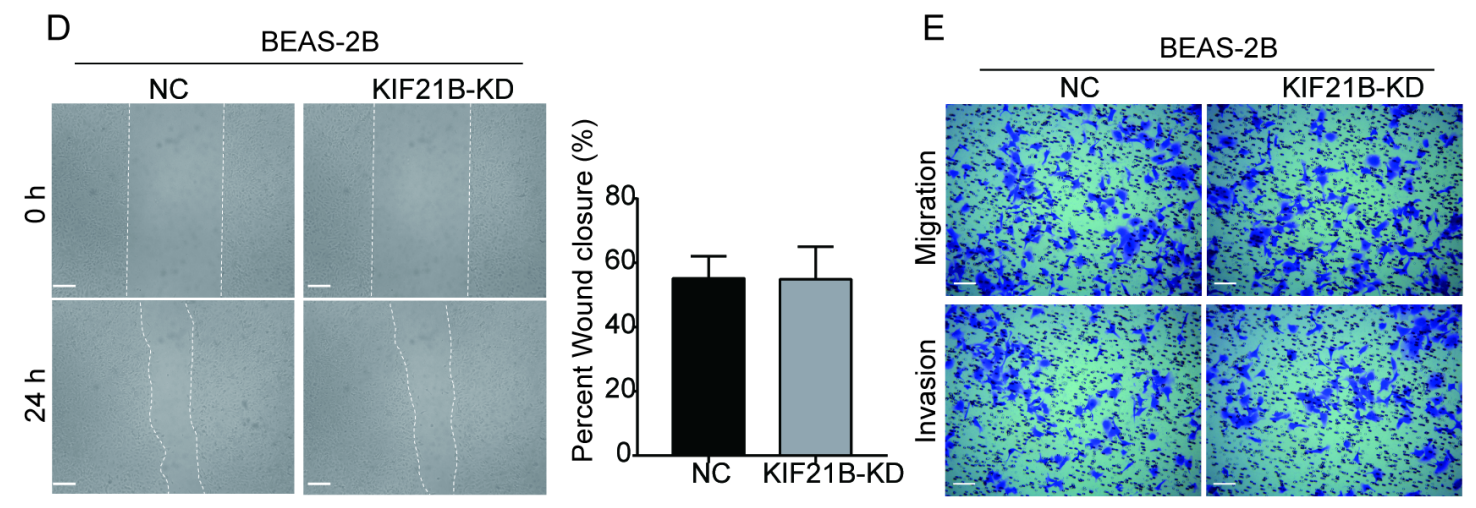
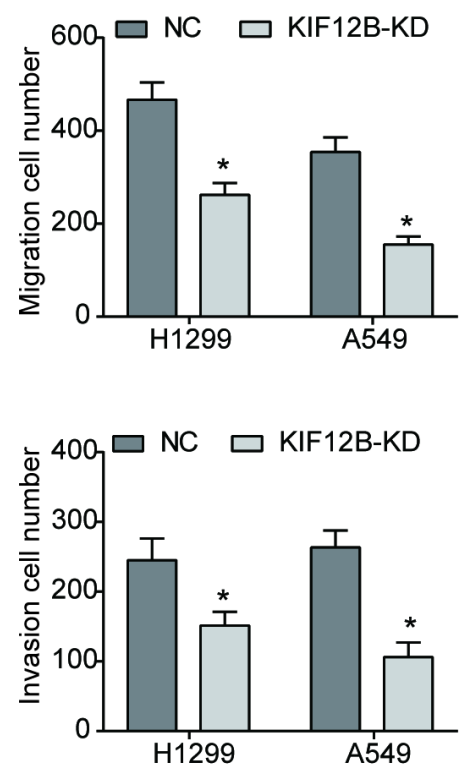

\section{Figure 5}

Silencing of KIF21B suppresses the migration and invasion abilities of NSCLC cells. (A) After being transfected with shRNAs, the effect of KIF21B knockdown on the migration of H1299 and A549 cells was assessed by wound-healing assay. (B) and (C) Transwell assay was performed for assessment of NSCLC cell migration (B) and invasion (C). (D) The effect of KIF21B knockdown on the migration of BEAS-2B cells was assessed by wound-healing assay. (E) Transwell assay was performed for assessment of the 
migration and invasion of BEAS-2B cells. Data are presented as the mean \pm standard deviation. Results were obtained in three replicates. The scale bar was $100 \mu \mathrm{m} .{ }^{*} \mathrm{P}<0.05$.

A

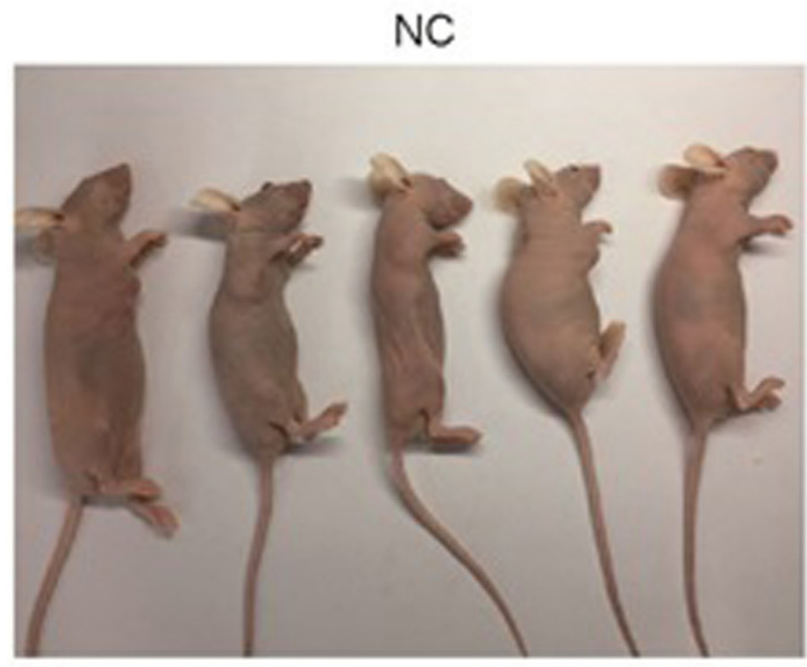

B

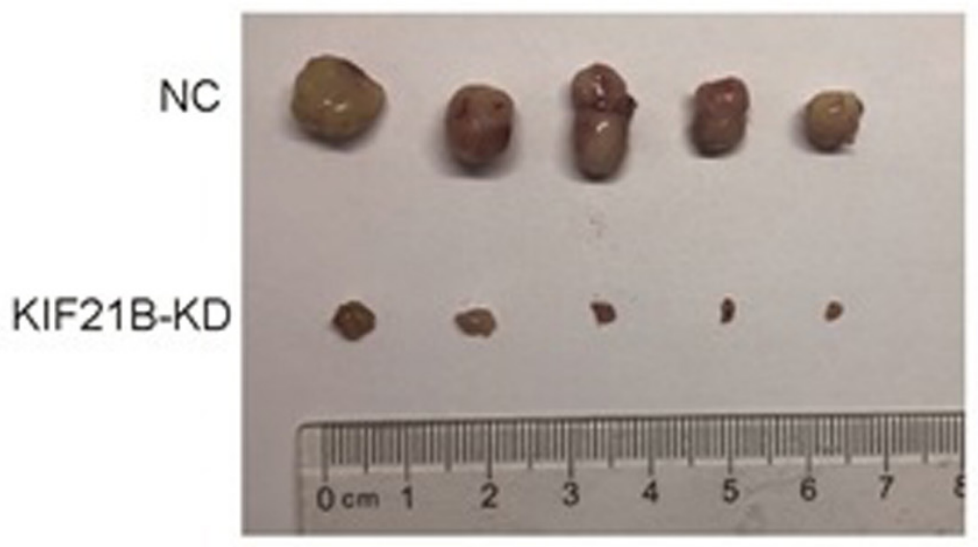

KIF21B-KD

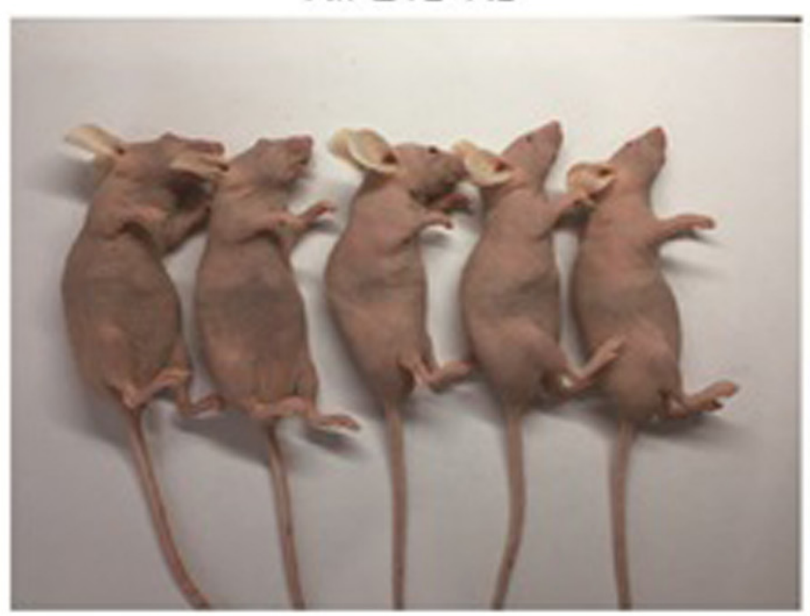

C

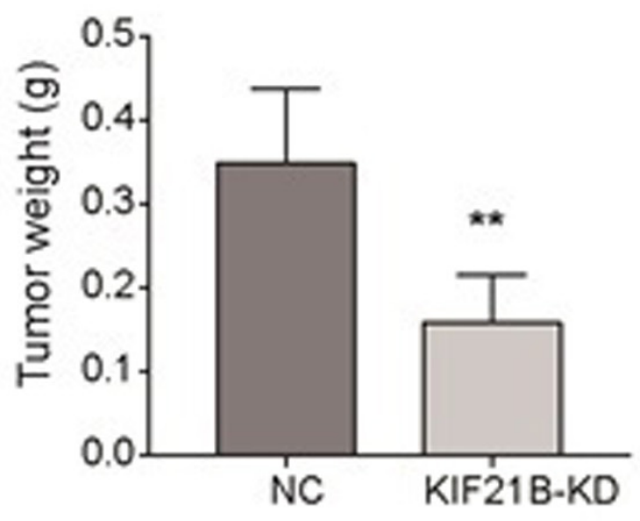

\section{Figure 6}

Knockdown of KIF21B inhibits tumor growth in nude mice. (A) The images of nude mice injected with H1299 cells transfected with shRNA-control lentivirus or sh-KIF21B lentivirus. (B and C) Representative xenograft tumors were shown (B) and the tumor weight was measured (C). ${ }^{\star *} \mathrm{P}<0.01$. 